\title{
The spatiotemporal transmission dynamics of COVID-19 among multiple regions: a modeling study in Chinese provinces
}

\author{
Qiaojuan Jia · Jiali Li · Hualiang Lin • \\ Fei Tian - Guanghu Zhu
}

Received: 22 July 2021 / Accepted: 14 October 2021 / Published online: 29 October 2021

(C) The Author(s), under exclusive licence to Springer Nature B.V. 2021

\begin{abstract}
Current explosive outbreak of COVID-19 around the world is a complex spatiotemporal process with hidden interactions between viruses and humans. This study aims at clarifying the transmission patterns and the driving mechanism that contributed to the COVID-19 prevalence across the provinces of China. Thus, a new dynamical transmission model is established by an ordinary differential system. The model takes into account the hidden circulation of COVID19 virus among/within humans, which incorporates the spatial diffusion of infection by parameterizing human mobility. Theoretical analysis indicates that the basic reproduction number is a unique epidemic threshold, which can unite infectivity in each region by human mobility and can totally determine whether COVID19 proceeds among multiple regions. By validating the model with real epidemic data in China, it is found that (1) if without any intervention, COVID-19 would overrun China within three months, resulting in more than 1.1 billion clinical infections and 0.2 billion subclinical infections; (2) high frequency of human mobility can
\end{abstract}

Supplementary Information The online version contains supplementary material available at https://doi.org/10.1007/ s11071-021-07001-1.

Q. Jia · J. Li · G. Zhu ( $\varangle)$

School of Mathematics and Computing Science, Guilin University of Electronic Technology, Guilin, China e-mail: ghzhu@guet.edu.cn

H. Lin · F. Tian

Department of Epidemiology, School of Public Health, Sun Yatsen University, Guangzhou 510080, China trigger COVID-19 diffusion across each province in China, no matter where the initial infection locates; (3) travel restrictions and other non-pharmaceutical interventions must be implemented simultaneously for disease control; and (4) infection sites in central and east (rather than west and northeast) of China would easily stimulate quick diffusion of COVID-19 in the whole country.

Keywords COVID-19 - Spatiotemporal transmission - Human mobility · Prevention and control . Reproduction number

\section{Introduction}

The pandemic coronavirus disease (COVID-19) is caused by a newly discovered coronavirus called SARS-CoV-2, which can spread from an infected person's mouth or nose in small liquid particles when they cough, sneeze, speak, sing or breathe [1]. Such easy transmission routes coupled with frequent human mobility quickly result in explosive outbreak around the world. As of September 22, 2021, this disease has attacked about 214 countries, with a total of over 230 million confirmed cases and over 4.7 million deaths [1]. COVID-19 is disrupting global health, economic, political, and social systems and is posing comprehensive threats to people around the world.

The ongoing COVID-19 pandemic exhibits a clear time-space evolution. As the first case was reported 
in Wuhan, China, on December 29, 2019, the disease has spread to all the provinces in China within a month [2]. By February 21, 2020, it has occurred in 27 countries, and the number of infected countries quickly increases to over 170 in late March. The infection size rose sharply from 282 to over 5 million during the 5-month period. Such fast diffusion and hierarchical structures in time and place were possibly shaped by human behaviors (e.g., communication, work, and movement). For example, after initial emergence in China, travel-related cases started appearing in other parts of the world with strong travel links to Wuhan [3]. This pattern along with the special characteristics of COVID-19: (1) high pathogenicity and hidden transmission among humans [4], (2) large asymptomatic patients with infectivity [5], (3) short serial interval [6], and (4) massive susceptibility [7], makes it very difficult to assess the risk and control the infection. Surveillance data indicate that the erupting infection of COVID-19 in China was quickly restrained due to the strict all-around interventions. Simulating its further potential progress in different circumstances and recognizing the spatiotemporal transmission dynamics can help to clarify the roles of the involved factors (e.g., human behavior, virus evolution, and intervention), identify the high-risk region, and guide the designing of targeted interventions in resource-limited settings. Yet little work is found in this regard.

Technically speaking, pure statistical model and mapping analysis can quantitatively tell the transmission pattern of epidemics. Mathematical frameworks incorporated epidemiological survey data can further capture its intrinsic variability in time and space, which are used increasingly in interdisciplinary studies [8]. Focusing on the COVID-19 pandemic, many epidemiology-inspired models, including SIR, SIS, and SEIR models, had been built to study the spreading patterns [9-14]. By simulating the underlying infection process, these studies found that (1) real-time mobility data from Wuhan can well elucidate the incidence rates in the cities across China [15]; (2) various non-pharmaceutical interventions are effective in controlling the spread of the disease [13,16-19]; and (3) mobility networks of air travel can predict the global diffusion pattern at the early stages of the outbreak, and an unconstrained mobility would have significantly accelerated COVID19 spread [20]. Due to the complexity and heterogeneity of COVID-19 diffusion, more efforts are needed to reveal its spatiotemporal dynamics [14, 21].

This paper goes a further step to provide a new modeling framework with consideration of human mobility and surveillance data to clarify the hidden dynamics accounting for COVID-19 spatiotemporal transmission in China. Based on the deterministic compartment model, a multi-population transmission model of COVID-19 is established by ordinary differential equations (ODE). Qualitative theory is used to analyze the propagation dynamics of the model, including the expression of the basic reproduction number and the equilibria, the global stability of the diseasefree and endemic equilibria. Finally, this model is applied to investigate the detailed transmission patterns of COVID-19 across the provinces in China.

\section{Modeling framework}

To simulate the spatiotemporal transmission of COVID19 across different regions, a new meta-population dynamic model is proposed in this section. Based on the epidemiology features of COVID-19 and compartmental theory, the following basic assumptions are proposed.

- During the outbreak of COVID-19 infection, humans are divided into susceptible $\left(S_{i}\right)$, exposed $\left(E_{i}\right)$, preclinical infectious $\left(I_{i}^{p}\right)$, subclinical infectious $\left(I_{i}^{S}\right)$, clinical infectious $\left(I_{i}^{c}\right)$ and recovered $\left(R_{i}\right)$ classes. Here $I_{i}^{p}$ and $I_{i}^{s}$ are inapparent infections, and $I_{i}^{c}$ are apparent infections. The sum of these classes constitute the total population size, that is, $N_{i}=S_{i}+E_{i}+I_{i}^{p}+I_{i}^{s}+I_{i}^{c}+R_{i}$. It is assumed that $N_{i}$ is a constant, in which birth rate equals to death rate $d$. Here, the subscript $i$ denotes the location of these parameters.

- The infection routes follow susceptible-latent -infected-recovered process. Individuals can be infected through contact with infectious individuals and then experience an incubation period $1 / \eta$. Exposed individuals progress to preclinical infectious (with probability $\phi$ ) and subclinical infectious (with probability $1-\phi$ ). Subclinical infections with mild or no symptoms could not be easily found and treated, but they can self-recover after time $1 / \gamma$. Preclinical infections become clinical and develop symptoms after time $1 / \delta$. They receive treatment and are cured successfully through time $1 / \omega$. 
- When novel coronavirus carried by infected humans invades into a virgin area, people there (local residents and visitors from other regions) could be infected with certain probability. The model takes into account such spatial diffusion by incorporating a migration matrix $P$, in which element $P_{i j}$ denotes the average duration per unit time that the residents in region $i$ stay in region $j$. It satisfies $P_{i j} \geq 0$ and $\sum_{j} P_{i j}=1$. Here residents can move around any other region, which may be infected outside and bring virus home. Specifically, due to human mobility, the real number of human population in region $j$ is $\tilde{N}_{j}=\sum_{k} P_{k j} N_{k}$, and the average proportions of preclinical, subclinical, clinical infections stay in region $j$ are $\sum_{k} P_{k j} I_{k}^{p} / \tilde{N}_{j}, \sum_{k} P_{k j} I_{k}^{s} / \tilde{N}_{j}$, and $\sum_{k} P_{k j} I_{k}^{c} / \tilde{N}_{j}$, respectively. Parts of susceptible residents of region $i$ (i.e., $P_{i j} S_{i}$ ) could be infected in region $j$ at rate $\lambda_{j}$.

Based on the above assumption, the essential features of the model framework are depicted in Fig. 1. Accordingly, the governing equations for simulating the spatiotemporal transmission dynamics of COVID-19 are illustrated as follows:

$$
\left\{\begin{aligned}
\frac{\mathrm{d} S_{i}}{\mathrm{~d} t}= & \mathrm{d} N_{i}-\sum_{j=1}^{n} \lambda_{j} P_{i j} S_{i} \\
& \times \frac{\sum_{k=1}^{n} P_{k j}\left(I_{k}^{p}+\alpha I_{k}^{s}+\beta I_{k}^{c}\right)}{\sum_{k=1}^{n} P_{k j} N_{k}}-\mathrm{d} S_{i}, \\
\frac{\mathrm{d} E_{i}}{\mathrm{~d} t}= & \sum_{j=1}^{n} \lambda_{j} P_{i j} S_{i} \frac{\sum_{k=1}^{n} P_{k j}\left(I_{k}^{p}+\alpha I_{k}^{s}+\beta I_{k}^{c}\right)}{\sum_{k=1}^{n} P_{k j} N_{k}} \\
& -(\eta+d) E_{i}, \\
\frac{\mathrm{d} I_{i}^{p}}{\mathrm{~d} t}= & \phi \eta E_{i}-(\delta+d) I_{i}^{p}, \\
\frac{\mathrm{d} I_{i}^{s}}{\mathrm{~d} t}= & (1-\phi) \eta E_{i}-(\gamma+d) I_{i}^{s}, \\
\frac{\mathrm{d} I_{i}^{c}}{\mathrm{~d} t}= & \delta I_{i}^{p}-(\omega+d) I_{i}^{c}, \\
\frac{\mathrm{d} R_{i}}{\mathrm{~d} t}= & \gamma I_{i}^{s}+\omega I_{i}^{c}-\mathrm{d} R_{i},
\end{aligned}\right.
$$

where $\lambda_{j}$ is the specific transmission rate in region $j$. The interpretation of other model parameters is presented in Table 1.

Since model (1) is a high-dimensional nonlinear ODE system, it is impossible to obtain its analyti- cal solution. To illustrate the long-term evolutions of model solutions and the robustness of these solutions to different initial conditions, the following two sections will explore the model behaviors mathematically, in which the basic reproduction number and the stability are discussed. By doing so, one can formulate the coupling pattern between disease transmission and human mobility and obtain the conditions under which the disease will die out or persist.

\section{Basic reproduction number}

The basic reproduction number $R_{0}$, as one of the most important theoretical concepts in epidemiology, acts as the critical measure of the transmissibility [23]. $R_{0}$ is interpreted as the average number of secondary cases that are produced by a single primary case in a fully susceptible population [23]. In what follows, it is written $\mathbf{S}=\left(S_{1}, S_{2}, \ldots, S_{n}\right)^{T}$ and similarly for $\mathbf{E}, \mathbf{I}^{\mathbf{p}}, \mathbf{I}^{\mathbf{s}}, \mathbf{I}^{\mathbf{c}}, \mathbf{R}$ and $\mathbf{N}$. Let $A_{p}$ be a $n \times n$ matrix, defined as

$$
A_{p}=\left(\begin{array}{ccc}
P_{11} & \cdots & P_{1 n} \\
\vdots & \ddots & \vdots \\
P_{n 1} & \cdots & P_{n n}
\end{array}\right)\left(\begin{array}{c}
\lambda_{1} \frac{P_{1}^{T}}{P_{1}^{T} \mathbf{N}} \\
\lambda_{2} \frac{P_{2}^{T}}{P_{2}^{T} \mathbf{N}} \\
\vdots \\
\lambda_{n} \frac{P_{n}^{T}}{P_{n}^{T} \mathbf{N}}
\end{array}\right),
$$

where the column vector $P_{i}$ is the i-th column of matrix $\left(P_{i j}\right)_{n \times n}$. Then, system (1) can then be rewritten as the following vectorial notation:

$$
\left\{\begin{aligned}
\frac{\mathrm{d} \mathbf{S}}{\mathrm{d} t}= & \mathrm{d} \mathbf{N}-\operatorname{diag}(\mathbf{S})\left(A_{p} \mathbf{I}^{\mathbf{p}}+\alpha A_{p} \mathbf{I}^{\mathbf{s}}+\beta A_{p} \mathbf{I}^{\mathbf{c}}\right) \\
& -\mathrm{d} \mathbf{S}, \\
\frac{\mathrm{d} \mathbf{E}}{\mathrm{d} t}= & \operatorname{diag}(\mathbf{S})\left(A_{p} \mathbf{I}^{\mathbf{p}}+\alpha A_{p} \mathbf{I}^{\mathbf{s}}+\beta A_{p} \mathbf{I}^{\mathbf{c}}\right) \\
& -(\eta+d) \mathbf{E} \\
\frac{\mathrm{d} \mathbf{I}^{\mathbf{p}}}{\mathrm{d} t}= & \phi \eta \mathbf{E}-(\delta+d) \mathbf{I}^{\mathbf{p}} \\
\frac{\mathrm{d} \mathbf{I}^{\mathbf{s}}}{\mathrm{d} t}= & (1-\phi) \eta \mathbf{E}-(\gamma+d) \mathbf{I}^{\mathbf{s}} \\
\frac{\mathrm{d} \mathbf{I}^{\mathbf{c}}}{\mathrm{d} t}= & \delta \mathbf{I}^{\mathbf{p}}-(\omega+d) \mathbf{I}^{\mathbf{c}} \\
\frac{\mathrm{d} \mathbf{R}}{\mathrm{d} t}= & \gamma \mathbf{I}^{\mathbf{s}}+\omega \mathbf{I}^{\mathbf{c}}-\mathrm{d} \mathbf{R} .
\end{aligned}\right.
$$


Fig. 1 Flow diagram on COVID-19 transmission with human mobility among different regions

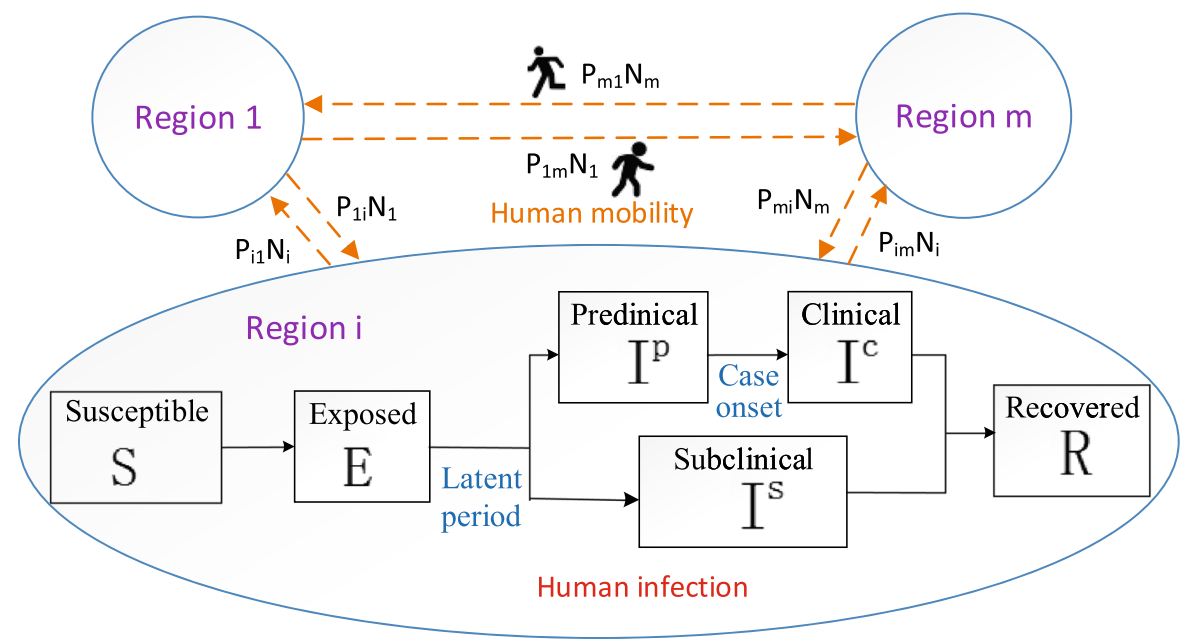

Table 1 Description of parameters in the proposed model

\begin{tabular}{llll}
\hline Parameters & Definitions & Value & Source \\
\hline$d$ & Birth/death rates & 0.0011 & Variable \\
$\alpha$ & Relative infectivity of subclinical infection & Variable & {$[\mathrm{b}]$} \\
$\beta$ & Relative infectivity of clinical infection & 4 days & {$[\mathrm{b}]$} \\
$1 / \eta$ & Duration of incubation period & 1.5 days & {$[17]$} \\
$1 / \delta$ & Time span of humans from preclinical onset to clinical patients & 14 days & {$[17]$} \\
$1 / \omega$ & Duration of treatment and recovery for clinical patients & 5 days & {$[1]$} \\
$1 / \gamma$ & Duration of recovery for subclinical infections & 0.82 & {$[17]$} \\
$\phi$ & Proportion of exposed individuals progress to preclinical infections & {$[22]$} &
\end{tabular}

[a] It is estimated from the data in China's National Bureau of Statistics in 2019

[b] These parameters are estimated by MCMC method in this study

Here for $u \in R^{n}, \operatorname{diag}(u)$ denotes the $n \times n$ diagonal matrix whose main diagonal is $u$. According to the biological significance, the initial values of model variables are set to be nonnegative, and then, the expressions of (2) can ensure that the solutions will always stay in

$$
\begin{aligned}
\Omega=\left\{\left(\mathbf{S}, \mathbf{E}, \mathbf{I}^{\mathbf{p}}, \mathbf{I}^{\mathbf{s}}, \mathbf{I}^{\mathbf{c}}, \mathbf{R}\right) \in R_{+}^{6 n} \mid 0\right. & \leq \mathbf{S}, \mathbf{E}, \mathbf{I}^{\mathbf{p}}, \mathbf{I}^{\mathbf{s}}, \mathbf{I}^{\mathbf{c}}, \mathbf{R} \\
& \leq \mathbf{N}\} .
\end{aligned}
$$

Hence, $\Omega$ is a compact absorbing and positively invariant set for (2). Direct calculation yields that system (2) has a disease-free equilibrium, denoted by $Q_{0}=$ $\left(\mathbf{S}^{\mathbf{0}}, \mathbf{E}^{\mathbf{0}}, \mathbf{I}^{\mathbf{p} \mathbf{0}}, \mathbf{I}^{\mathbf{s} \mathbf{0}}, \mathbf{I}^{\mathbf{c} \mathbf{0}}, \mathbf{R}^{\mathbf{0}}\right)=(\mathbf{N}, \mathbf{0}, \mathbf{0}, \mathbf{0}, \mathbf{0}, \mathbf{0})$.

The basic reproduction number $R_{0}$ is calculated by using the theory of next-generation matrix [23]. It is written as $R_{0}=\rho\left(\mathrm{FV}^{-1}\right)$, where $F$ is the rate of occur- ring new infections, and $V$ is the rate of transferring individuals outside the original group [23]. Here $\rho$ represents the spectral radius of matrix. Based on model (1), direct calculation yields that:

$F=\left(\begin{array}{cccc}\mathbf{O} & \operatorname{diag}(\mathbf{N}) A_{p} & \alpha \operatorname{diag}(\mathbf{N}) A_{p} & \beta \operatorname{diag}(\mathbf{N}) A_{p} \\ \mathbf{O} & \mathbf{O} & \mathbf{O} & \mathbf{O} \\ \mathbf{O} & \mathbf{O} & \mathbf{O} & \mathbf{O} \\ \mathbf{O} & \mathbf{O} & \mathbf{O} & \mathbf{O}\end{array}\right)$

and

$V=\left(\begin{array}{cccc}(\eta+d) \mathbf{I} & \mathbf{O} & \mathbf{O} & \mathbf{O} \\ -\phi \eta \mathbf{I} & (\delta+d) \mathbf{I} & \mathbf{O} & \mathbf{O} \\ -(1-\phi) \eta \mathbf{I} & \mathbf{O} & (\gamma+d) \mathbf{I} & \mathbf{O} \\ \mathbf{O} & -\delta \mathbf{I} & \mathbf{O} & (\omega+d) \mathbf{I}\end{array}\right)$.

where $\mathbf{I}$ denotes a identity matrix, and $\mathbf{O}$ is the zero matrix. It follows from the characteristic equation of $F V^{-1}$ that the basic reproduction number is given by: 


$$
\begin{aligned}
R_{0}= & \rho\left(\left(\frac{\phi \eta}{(\eta+d)(\delta+d)}+\frac{(1-\phi) \eta \alpha}{(\eta+d)(\gamma+d)}\right.\right. \\
& \left.\left.+\frac{\phi \eta \delta \beta}{(\eta+d)(\delta+d)(\omega+d)}\right) \operatorname{diag}(\mathbf{N}) A_{p}\right) .
\end{aligned}
$$

The three components of the $R_{0}$ are separately contributed by the infections in preclinical, subclinical, and clinical states. Since the characteristic equation in (3) is a polynomial of degree $n$ for eigenvalue, it is impossible to calculate its analytic expression.

\section{Global stability}

The results concerning the global dynamics of system (2) are analyzed in this section.

Theorem 4.1 System (2) has a unique endemic equilibrium $Q^{*}$.

Proof It is denoted as the expression of endemic equilibrium by $\mathbf{S}^{*}, \mathbf{E}^{*}, \mathbf{I}^{\mathbf{p} *}, \mathbf{I}^{\mathbf{S} *}$ and $\mathbf{I}^{\mathbf{c} *}$. Based on the equilibrium definition, letting the right-hand side of system (2) to be zeros and substituting $\mathbf{S}^{*}, \mathbf{E}^{*}, \mathbf{I}^{\mathbf{S} *}$, and $\mathbf{I}^{\mathbf{*} *}$ by $\mathbf{I}^{\mathbf{p} *}$, an equation is obtained about $\mathbf{I}^{\mathbf{p} *}$ as

$$
\begin{aligned}
f\left(\mathbf{I}^{\mathbf{p} *}\right)= & m_{1} \mathbf{I}^{\mathbf{p} *}\left(\operatorname{diag}\left(m_{2} A_{p} \mathbf{I}^{\mathbf{p} *}\right)\right)^{-1}\left(\mathrm{~d} \mathbf{I}+m_{2} A_{p} \mathbf{I}^{\mathbf{p} *}\right) \\
& -\mathrm{d} \mathbf{N},
\end{aligned}
$$

where

$$
\begin{aligned}
& m_{1}=\frac{(\eta+d)(\delta+d)}{\phi \eta}, \\
& m_{2}=1+\frac{\alpha(1-\phi)(\delta+d)}{\phi(\gamma+d)}+\frac{\beta \delta}{\omega+d} .
\end{aligned}
$$

Substituting $\mathbf{I}^{\mathbf{p} *}$ by $\mathbf{0}$ and $\mathbf{N}$ yields that $f(\mathbf{0})=-\mathrm{d} \mathbf{N}<$ 0, and

$$
\begin{aligned}
f(\mathbf{N})= & m_{1} \mathbf{N}\left(\operatorname{diag}\left(m_{2} A_{p} \mathbf{N}\right)\right)^{-1}\left(d \mathbf{1}+m_{2} A_{p} \mathbf{N}\right)-\mathrm{d} \mathbf{N} \\
\geq & \frac{(\delta+d)}{\phi} \mathbf{N}\left(\operatorname{diag}\left(A_{p} \mathbf{N}\right)\right)^{-1}\left(A_{p} \mathbf{N}\right) \\
& -\mathrm{d} \mathbf{N}=\frac{(\delta+d)}{\phi} \mathbf{N}-\mathrm{d} \mathbf{N}>\mathbf{0} .
\end{aligned}
$$

It follows from the zero-point theorem that system (2) has at least one positive equilibrium. Furthermore, due to $f^{\prime}\left(\mathbf{I}^{\mathbf{p}}\right)=m_{1} \mathbf{I}>\mathbf{0}, f$ is an increasing function. Hence, there exists a unique positive endemic equilibrium in the compact set $\Omega$.

Theorem 4.2 If $R_{0}<1$, the disease-free equilibrium $Q_{0}$ of system (2) is globally asymptotically stable.
Proof Since the total number of human population is a constant, the first equation of system (2) can be ignored. Substituting $\mathbf{S}$ by $\left(\mathbf{N}-\mathbf{E}-\mathbf{I}^{\mathbf{p}}-\mathbf{I}^{\mathbf{S}}-\mathbf{I}^{\mathbf{c}}-\mathbf{R}\right)$, it is obtained

$$
\begin{aligned}
& \frac{\mathrm{d} \mathbf{E}}{\mathrm{d} t}=\operatorname{diag}\left(\mathbf{N}-\mathbf{E}-\mathbf{I}^{\mathbf{p}}-\mathbf{I}^{\mathbf{s}}-\mathbf{I}^{\mathbf{c}}-\mathbf{R}\right) \\
& \quad\left(A_{p} \mathbf{I}^{\mathbf{p}}+\alpha A_{p} \mathbf{I}^{\mathbf{s}}+\beta A_{p} \mathbf{I}^{\mathbf{c}}\right)-(\eta+d) \mathbf{E} \\
& \leq \operatorname{diag}(\mathbf{N})\left(A_{p} \mathbf{I}^{\mathbf{p}}+\alpha A_{p} \mathbf{I}^{\mathbf{s}}+\beta A_{p} \mathbf{I}^{\mathbf{c}}\right)-(\eta+d) \mathbf{E} .
\end{aligned}
$$

The corresponding comparison system is:

$$
\left\{\begin{aligned}
\frac{\mathrm{d} \overline{\mathbf{E}}}{\mathrm{d} t}= & \operatorname{diag}(\mathbf{N})\left(A_{p} \overline{\mathbf{I}}^{\mathbf{p}}+\alpha A_{p} \overline{\mathbf{I}}^{\mathbf{s}}+\beta A_{p} \overline{\mathbf{I}}^{\mathbf{c}}\right) \\
& -(\eta+d) \overline{\mathbf{E}}, \\
\frac{\mathrm{d} \overline{\mathbf{I}}^{\mathbf{p}}}{\mathrm{d} t}= & \phi \eta \overline{\mathbf{E}}-(\delta+d) \overline{\mathbf{I}} \mathbf{p}, \\
\frac{\mathrm{d} \overline{\mathbf{I}}^{\mathbf{s}}}{\mathrm{d} t}= & (1-\phi) \eta \overline{\mathbf{E}}-(\gamma+d) \overline{\mathbf{I}}^{\mathbf{s}}, \\
\frac{\mathrm{d} \overline{\mathbf{I}}^{\mathbf{c}}}{\mathrm{d} t}= & \delta \overline{\mathbf{I}}^{\mathbf{p}}-(\omega+d) \overline{\mathbf{I}}^{\mathbf{c}} .
\end{aligned}\right.
$$

It is clear that model (4) is a linear system, and the coefficient matrix of its variables in the righthand side is exactly the matrix $(F-V)$. Hence, when $R_{0}=\rho\left(F V^{-1}\right)<1$, the unique equilibrium $\left(\mathbf{E}, \mathbf{I}^{\mathbf{p}}, \mathbf{I}^{\mathbf{s}}, \mathbf{I}^{\mathbf{c}}\right)=(\mathbf{0}, \mathbf{0}, \mathbf{0}, \mathbf{0})$ of this linear system (4) is globally asymptotically stable. Since

$\frac{\mathrm{d} \mathbf{E}}{\mathrm{d} t} \leq \frac{d \overline{\mathbf{E}}}{\mathrm{d} t}, \frac{\mathrm{d} \mathbf{I}^{\mathbf{p}}}{\mathrm{d} t} \leq \frac{\mathrm{d} \overline{\mathbf{I}}^{\mathbf{p}}}{\mathrm{d} t}, \frac{\mathrm{d} \mathbf{I}^{\mathbf{s}}}{\mathrm{d} t} \leq \frac{\mathrm{d} \overline{\mathbf{I}^{\mathbf{s}}}}{\mathrm{d} t}, \frac{\mathrm{d} \mathbf{I}^{\mathbf{c}}}{\mathrm{d} t} \leq \frac{\mathrm{d} \overline{\mathbf{I}^{\mathbf{c}}}}{\mathrm{d} t}$.

According to the comparison theorem, with the same initial conditions, it has $\mathbf{E}(t) \leq \overline{\mathbf{E}}(t), \mathbf{I}^{p}(t) \leq \overline{\mathbf{I}}^{p}(t)$, $\mathbf{I}^{s}(t) \leq \overline{\mathbf{I}}^{s}(t)$, and $\mathbf{I}^{c}(t) \leq \overline{\mathbf{I}}^{c}(t)$ for any $t>0$, yielding that $Q_{0}$ is globally asymptotically stable when $R_{0}<1$.

The graph-theoretic method presented in [24,25] is used to analyze the global stability of the endemic equilibrium.

Theorem 4.3 If $R_{0}>1$, then the unique endemic equilibrium $Q^{*}$ of system (2) is globally asymptotically stable in $\Omega$.

Proof Denote

$$
\begin{aligned}
D_{i} & =S_{i}-S_{i}^{*}-S_{i}^{*} \ln \frac{S_{i}}{S_{i}^{*}}+E_{i}-E_{i}^{*}-E_{i}^{*} \ln \frac{E_{i}}{E_{i}^{*}}, \\
D_{n+i} & =I_{i}^{p}-I_{i}^{p *}-I_{i}^{p *} \ln \frac{I_{i}^{p}}{I_{i}^{p *}},
\end{aligned}
$$


$D_{2 n+i}=I_{i}^{s}-I_{i}^{s *}-I_{i}^{s *} \ln \frac{I_{i}^{s}}{I_{i}^{s *}}$,

$D_{3 n+i}=I_{i}^{c}-I_{i}^{c *}-I_{i}^{c *} \ln \frac{I_{i}^{c}}{I_{i}^{c *}}, \tilde{N}_{j}=\sum_{k=1}^{n} P_{k j} N_{k}$,

where the variables with superscript as star are the expressions of endemic equilibrium in model. Using the inequality $1-x+\ln x \leq 0$, for $x>0$, direct differentiation yields:

$$
\begin{aligned}
& D_{i}{ }^{\prime}=\sum_{j=1}^{n} \lambda_{j} P_{i j} S_{i}^{*} \ell_{j}{ }^{*}+\mathrm{d} S_{i}^{*}-\sum_{j=1}^{n} \lambda_{j} P_{i j} S_{i} \ell_{j} \\
& -\mathrm{d} S_{i}-\sum_{j=1}^{n} \lambda_{j} P_{i j} S_{i}^{*} \frac{S_{i}^{*}}{S_{i}} \ell_{j}^{*}-d \frac{S_{i}^{*} S_{i}^{*}}{S_{i}} \\
& +\sum_{j=1}^{n} \lambda_{j} P_{i j} S_{i}^{*} \ell_{j}+\mathrm{d} S_{i}^{*}+\sum_{j=1}^{n} \lambda_{j} P_{i j} S_{i} \ell_{j} \\
& -\sum_{j=1}^{n} \lambda_{j} P_{i j} S_{i}^{*} \frac{E_{i}}{E_{i}^{*}} \ell_{j}^{*}-\sum_{j=1}^{n} \lambda_{j} P_{i j} S_{i} \frac{E_{i}^{*}}{E_{i}} \ell_{j} \\
& +\sum_{j=1}^{n} \lambda_{j} P_{i j} S_{i}^{*} \ell_{j}^{*} \\
& \leq \sum_{j=1}^{n} \lambda_{j} P_{i j} S_{i}^{*} \ell_{j}^{*}-\sum_{j=1}^{n} \lambda_{j} P_{i j} S_{i} \ell_{j} \\
& -\sum_{j=1}^{n} \lambda_{j} P_{i j} S_{i}^{*} \frac{S_{i}^{*}}{S_{i}} \ell_{j}^{*}+\sum_{j=1}^{n} \lambda_{j} P_{i j} S_{i}^{*} \ell_{j} \\
& +\sum_{j=1}^{n} \lambda_{j} P_{i j} S_{i}-\sum_{j=1}^{n} \lambda_{j} P_{i j} S_{i}^{*} \frac{E_{i}}{E_{i}^{*}} \ell_{j}{ }^{*} \\
& -\sum_{j=1}^{n} \lambda_{j} P_{i j} S_{i} \frac{E_{i}^{*}}{E_{i}} \ell_{j}+\sum_{j=1}^{n} \lambda_{j} P_{i j} S_{i}^{*} \ell_{j}{ }^{*} \\
& =\sum_{j=1}^{n} \lambda_{j} P_{i j} S_{i}^{*} \varpi_{j}^{p}\left(1-\frac{S_{i} I_{k}^{p}}{S_{i}^{*} I_{k}^{p *}}-\frac{S_{i}^{*}}{S_{i}}+\frac{I_{k}^{p}}{I_{k}^{p *}}\right) \\
& +\sum_{j=1}^{n} \lambda_{j} P_{i j} S_{i}^{*} \alpha \varpi_{j}^{s}\left(1-\frac{S_{i} I_{k}^{s}}{S_{i}^{*} I_{k}^{s *}}-\frac{S_{i}^{*}}{S_{i}}+\frac{I_{k}^{s}}{I_{k}^{s *}}\right) \\
& +\sum_{j=1}^{n} \lambda_{j} P_{i j} S_{i}^{*} \beta \varpi_{j}^{c}\left(1-\frac{S_{i} I_{k}^{c}}{S_{i}^{*} I_{k}^{c *}}-\frac{S_{i}^{*}}{S_{i}}+\frac{I_{k}^{c}}{I_{k}^{c *}}\right) \\
& +\sum_{j=1}^{n} \lambda_{j} P_{i j} S_{i}^{*} \varpi_{j}^{p}\left(1+\frac{S_{i} I_{k}^{p}}{S_{i}^{*} I_{k}^{p *}}-\frac{E_{i}}{E_{i}^{*}}-\frac{E_{i}^{*} S_{i} I_{k}^{p}}{E_{i} S_{i}^{*} I_{k}^{p *}}\right) \\
& +\sum_{j=1}^{n} \lambda_{j} P_{i j} S_{i}^{*} \alpha \varpi_{j}^{s}\left(1+\frac{S_{i} I_{k}^{s}}{S_{i}^{*} I_{k}^{s *}}-\frac{E_{i}}{E_{i}^{*}}-\frac{E_{i}^{*} S_{i} I_{k}^{s}}{E_{i} S_{i}^{*} I_{k}^{s *}}\right)
\end{aligned}
$$

$$
\begin{aligned}
& +\sum_{j=1}^{n} \lambda_{j} P_{i j} S_{i}^{*} \beta \varpi_{j}^{c}\left(1+\frac{S_{i} I_{k}^{c}}{S_{i}^{*} I_{k}^{c *}}-\frac{E_{i}}{E_{i}^{*}}-\frac{E_{i}^{*} S_{i} I_{k}^{c}}{E_{i} S_{i}^{*} I_{k}^{c *}}\right) \\
\leq & \sum_{j=1}^{n} \lambda_{j} P_{i j} S_{i}^{*} \varpi_{j}^{p}\left(\frac{I_{k}^{p}}{I_{k}^{p *}}-\ln \frac{I_{k}^{p}}{I_{k}^{p *}}+\ln \frac{E_{i}}{E_{i}^{*}}-\frac{E_{i}}{E_{i}^{*}}\right) \\
& +\sum_{j=1}^{n} \lambda_{j} P_{i j} S_{i}^{*} \alpha \varpi_{j}^{s}\left(\frac{I_{k}^{s}}{I_{k}^{s *}}-\ln \frac{I_{k}^{s}}{I_{k}^{s *}}+\ln \frac{E_{i}}{E_{i}^{*}}-\frac{E_{i}}{E_{i}^{*}}\right) \\
& +\sum_{j=1}^{n} \lambda_{j} P_{i j} S_{i}^{*} \beta \varpi_{j}^{c}\left(\frac{I_{k}^{c}}{I_{k}^{c *}}-\ln \frac{I_{k}^{c}}{I_{k}^{c *}}+\ln \frac{E_{i}}{E_{i}^{*}}-\frac{E_{i}}{E_{i}^{*}}\right) \\
= & : a_{i, n+i} G_{i, n+i}+a_{i, 2 n+i} G_{i, 2 n+i}+a_{i, 3 n+i} G_{i, 3 n+i} .
\end{aligned}
$$

Here,

$$
\begin{aligned}
\ell_{j}^{*} & =\frac{1}{\tilde{N}_{j}} \sum_{k=1}^{n} P_{k j}\left(I_{k}^{p *}+\alpha I_{k}^{s *}+\beta I_{k}^{c *}\right), \\
\ell_{j} & =\frac{1}{\tilde{N}_{j}} \sum_{k=1}^{n} P_{k j}\left(I_{k}^{p}+\alpha I_{k}^{s}+\beta I_{k}^{c}\right), \\
\varpi_{j}^{p} & =\frac{1}{\tilde{N}_{j}} \sum_{k=1}^{n} P_{k j} I_{k}^{p *}, \\
\varpi_{j}^{s} & =\frac{1}{\tilde{N}_{j}} \sum_{k=1}^{n} P_{k j} I_{k}^{s *}, \varpi_{j}^{c}=\frac{1}{\tilde{N}_{j}} \sum_{k=1}^{n} P_{k j} I_{k}^{c *} .
\end{aligned}
$$

Similarly,

$$
\begin{aligned}
D^{\prime}{ }_{n+i}= & \phi \eta E_{i}-\phi \eta E_{i}^{*} \frac{I_{i}^{p}}{I_{i}^{p *}}-\phi \eta E_{i} \frac{I_{i}^{p *}}{I_{i}^{p}}+\phi \eta E_{i}^{*} \\
= & \phi \eta E_{i}^{*}\left(1-\frac{E_{i} I_{i}^{p *}}{E_{i}^{*} I_{i}^{p}}-\frac{I_{i}^{p}}{I_{i}^{p *}}+\frac{E_{i}}{E_{i}^{*}}\right) \\
\leq & \phi \eta E_{i}^{*}\left(\frac{E_{i}}{E_{i}^{*}}-\ln \frac{E_{i}}{E_{i}^{*}}+\ln \frac{I_{i}^{p}}{I_{i}^{p *}}-\frac{I_{i}^{p}}{I_{i}^{p *}}\right) \\
= & : a_{n+i, i} G_{n+i, i} . \\
D_{2 n+i}^{\prime}= & (1-\phi) \eta E_{i}-(1-\phi) \eta E_{i}^{*} \frac{I_{i}^{s}}{I_{i}^{s *}} \\
& -(1-\phi) \eta E_{i} \frac{I_{i}^{s *}}{I_{i}^{s}}+(1-\phi) \eta E_{i}^{*} \\
= & (1-\phi) \eta E_{i}^{*}\left(1-\frac{E_{i} I_{i}^{s *}}{E_{i}^{*} I_{i}^{s}}-\frac{I_{i}^{s}}{I_{i}^{s *}}+\frac{E_{i}}{E_{i}^{*}}\right) \\
\leq & (1-\phi) \eta E_{i}^{*}\left(\frac{E_{i}}{E_{i}^{*}}-\ln \frac{E_{i}}{E_{i}^{*}}+\ln \frac{I_{i}^{s}}{I_{i}^{s *}}-\frac{I_{i}^{s}}{I_{i}^{s *}}\right) \\
= & : a_{2 n+i, i} G_{2 n+i, i} \cdot \\
D_{3 n+i}^{\prime}= & \delta I_{i}^{p}-\delta I_{i}^{p *} \frac{I_{i}^{c}}{I_{i}^{c *}}-\delta I_{i}^{p} \frac{I_{i}^{s *}}{I_{i}^{s}}+\delta I_{i}^{p *}
\end{aligned}
$$




$$
\begin{aligned}
& =\delta I_{i}^{p *}\left(1-\frac{I_{i}^{p} I_{i}^{c *}}{I_{i}^{p *} I_{i}^{c}}-\frac{I_{i}^{c}}{I_{i}^{c *}}+\frac{I_{i}^{p}}{I_{i}^{p *}}\right) \\
& \leq \delta I_{i}^{p *}\left(\frac{I_{i}^{p}}{I_{i}^{p *}}-\ln \frac{I_{i}^{p}}{I_{i}^{p *}}+\ln \frac{I_{i}^{c}}{I_{i}^{c *}}-\frac{I_{i}^{c}}{I_{i}^{c *}}\right) \\
& =: a_{3 n+i, n+i} G_{3 n+i, n+i} .
\end{aligned}
$$

and

$$
\begin{aligned}
a_{i, n+i} & =\frac{\lambda_{j} P_{i j} S_{i}^{*}}{\tilde{N}_{j}} \sum_{k=1}^{n} P_{k j} I_{k}^{p *}, \\
a_{i, 2 n+i} & =\frac{\lambda_{j} P_{i j} S_{i}^{*} \alpha}{\tilde{N}_{j}} \sum_{k=1}^{n} P_{k j} I_{k}^{s *}, \\
a_{i, 3 n+i} & =\frac{\lambda_{j} P_{i j} S_{i}^{*} \beta}{\tilde{N}_{j}} \sum_{k=1}^{n} P_{k j} I_{k}^{c *},
\end{aligned}
$$

as well as $a_{n+i, i}=\phi \eta E_{i}^{*}, a_{2 n+i, i}=(1-\phi) \eta E_{i}^{*}$, $a_{3 n+i, i}=\delta I_{i}^{p *}$. Let $A=\left(a_{i j}\right)_{n \times n}$ with $a_{i j}>0$ as defined above and otherwise zero. The corresponding weighted digraph is shown in Fig. 2. Along each of the cycles on the graph, it is verified that $\sum G_{i j}=0$; for instance, $G_{i, n+i}+G_{n+i, i}=0, G_{j, n+i}+G_{n+j, j}+$ $G_{i, n+j}+G_{n+i, i}=0$, and so on. It follows from Theorem 3.5 in [24] that there exist constants $c_{i}$ such that $D=\sum_{i} c_{i} D_{i}$ is a Lyapunov function for system (2).

Let $c_{1}=\cdots=c_{n}=1$, and

$$
\begin{aligned}
c_{n+i} & =\sum_{j=1}^{n} \frac{\left(c_{j} a_{j, n+i}+c_{j} a_{j, 3 n+i}\right)}{a_{n+i, i}}, \\
c_{2 n+i} & =\sum_{j=1}^{n} \frac{c_{j} a_{j, 2 n+i}}{a_{2 n+i, i}}, c_{3 n+i}=\sum_{j=1}^{n} \frac{c_{j} a_{j, 3 n+i}}{a_{3 n+i, n+i}} .
\end{aligned}
$$

Further computation leads to

$$
\begin{aligned}
c_{n+i}= & \sum_{j=1}^{n}\left[\frac { \lambda _ { j } P _ { i j } S _ { i } ^ { * } } { \tilde { N } _ { j } \phi \eta E _ { i } ^ { * } } \left(\sum_{k=1}^{n} P_{k j} I_{k}^{p *}\right.\right. \\
& \left.\left.+\beta \sum_{k=1}^{n} P_{k j} I_{k}^{c *}\right)\right], \\
c_{2 n+i}= & \sum_{j=1}^{n}\left(\frac{\lambda_{j} P_{i j} S_{i}^{*} \alpha}{(1-\phi) \eta E_{i}^{*} \tilde{N}_{j}} \sum_{k=1}^{n} P_{k j} I_{k}^{s *}\right), \\
c_{3 n+i}= & \sum_{j=1}^{n}\left(\frac{\lambda_{j} P_{i j} S_{i}^{*} \beta}{\delta I_{i}^{p *} \tilde{N}_{j}} \sum_{k=1}^{n} P_{k j} I_{k}^{c *}\right) .
\end{aligned}
$$

Hence, with the functions $D_{i}$ and constants $c_{i}$ given above, the expression

$$
D=\sum_{i=1}^{n} c_{i} D_{i}+\sum_{i=1}^{n} c_{n+i} D_{n+i}
$$

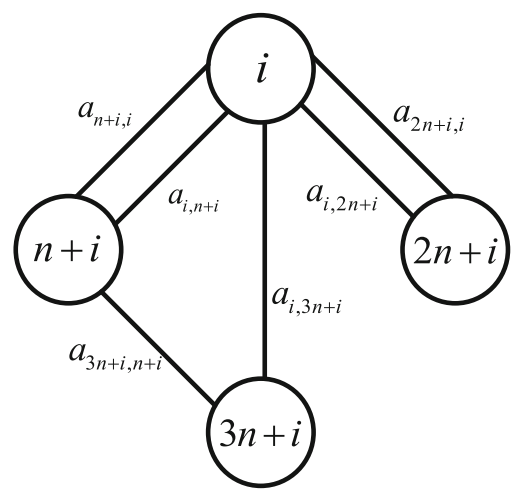

Fig. 2 Digraph representation of the matrix A of transmission used to determine the coefficients in the Lyapunov function D

$$
+\sum_{i=1}^{n} c_{2 n+i} D_{2 n+i}+\sum_{i=1}^{n} c_{3 n+i} D_{3 n+i}
$$

is a Lyapunov function for system (2). Its derivative is:

$$
\begin{aligned}
D^{\prime}= & \sum_{i=1}^{n} c_{i}\left(\frac{S_{i}-S_{i}^{*}}{S_{i}} S_{i}{ }^{\prime}+\frac{E_{i}-E_{i}^{*}}{E_{i}} E_{i}{ }^{\prime}\right) \\
& +\sum_{i=1}^{n} c_{n+i}\left(\frac{I_{i}^{p}-I_{i}^{p *}}{I_{i}^{p}} I_{i}^{p^{\prime}}\right) \\
& +\sum_{i=1}^{n} c_{2 n+i}\left(\frac{I_{i}^{s}-I_{i}^{s *}}{I_{i}^{s}} I_{i}^{s^{\prime}}\right) \\
& +\sum_{i=1}^{n} c_{3 n+i}\left(\frac{I_{i}^{c}-I_{i}^{c *}}{I_{i}^{c}} I_{i}^{c^{\prime}}\right) .
\end{aligned}
$$

When $D^{\prime}=0$ in the set $\left\{R_{+}^{5 n}\right\}$, one can readily verify that $S_{i}=S_{i}^{*}, E_{i}=E_{i}^{*}, I_{i}^{p}=I_{i}^{p *}, I_{i}^{s}=I_{i}^{s *}, I_{i}^{c}=$ $I_{i}^{c *}$. For the left system,

$\frac{d R_{i}}{\mathrm{~d} t}=\gamma I_{i}^{s *}+\omega I_{i}^{c *}-d R_{i}$.

it is clear that system (2) has a unique equilibrium $R_{i}=R_{i}^{*}$, which is global asymptotically stable. Using LaSalle's invariance principle, it is concluded that the endemic equilibrium $Q^{*}$ is global asymptotically stable in $\Omega$.

\section{Application to the outbreak in China}

In this section, the proposed model is applied to analyze the spatiotemporal transmission dynamics of COVID19 in Chinese provinces (see Table 2). Daily records of human infections were collected from authoritative 
Table 2 Province and its abbreviation in China

\begin{tabular}{|c|c|c|c|c|c|c|c|c|}
\hline Province & Hubei & Beijing & tianjin & Hebei & Shanxi & Liaoning & Jilin & Heilongjiang \\
\hline Abbreviation & $\mathrm{HuB}$ & $\mathrm{BJ}$ & TJ & $\mathrm{HeB}$ & SX & $\mathrm{LN}$ & $\mathrm{JL}$ & HLJ \\
\hline Province & Shanghai & Jiangsu & Zhejiang & Anhui & Fujian & Jiangxi & Shandong & Henan \\
\hline Abbreviation & $\mathrm{SH}$ & JS & $\mathrm{ZJ}$ & $\mathrm{AH}$ & FJ & $\mathrm{JX}$ & $\mathrm{SD}$ & $\mathrm{HeN}$ \\
\hline Province & Hunan & Guangdong & Guangxi & Hainan & Chongqing & Sichuan & Guizhou & Yunnan \\
\hline Abbreviation & $\mathrm{HuN}$ & GD & GX & HaiN & CQ & $\mathrm{SC}$ & GZ & YN \\
\hline Province & Shaanxi & Gansu & Qinghai & Ningxia & Xinjiang & Neimenggu & Xizang & \\
\hline Abbreviation & $\mathrm{SaX}$ & GS & $\mathrm{QH}$ & $\mathrm{NX}$ & $\mathrm{XJ}$ & NMG & $\mathrm{XZ}$ & \\
\hline
\end{tabular}

data report. The permanent population size in each province was released by the 2019 National Bureau of Statistics. The daily migration data among provinces are collected from Baidu migration data (https://qianxi. baidu.com/). Specifically, the element in migration matrix $P$ is defined as $P_{i j}=\kappa Q_{i} c_{i j}$, where $Q_{i}$ is the migration scale in region $i$ and $c_{i j}$ is the proportion of migration scale from region $i$ to region $j$ (both of which were extracted from Baidu migration data), and $\kappa$ is a adjustive constant for modulating the data into the model.

The model is validated by using Markov chain Monte Carlo (MCMC) method to fit the daily reported cases in 26 provinces (with cases more than 101 from January 5, 2020, to March 15, 2020). Here 6 parameters $\left(\beta, \alpha, \kappa\right.$, and the initial values of $E, I^{c}$ and $I^{p}$ in $\left.\mathrm{HuB}\right)$ were estimated by MCMC. Since HuB province is considered to be the infection source, it is assumed that there is no infections in other provinces at initial time. The transmission rate $\lambda_{i}$ is derived from the effective reproduction number $R_{t}$ in province $i$. $R_{t}$ represents the number of new morbidity cases caused by an average morbidity case at time $t$. Here the $R_{t}$ in each province is estimated from the time series of its indigenous cases. Based on the Bayesian framework, $R_{t}$ is calculated by the EpiEstim package in $\mathrm{R}$ language software [26], in which the intergenerational time follows gamma distribution, with the mean value and standard deviation as 7.5 and 3.4, respectively [27].

The fitting results are shown in Figs. 3 and S1 (in Supplementary Information). It is found that the model performed well in fitting the daily reported incidences, except the data in some provinces such as $\mathrm{HeB}, \mathrm{ZJ}$, $\mathrm{HeN}, \mathrm{HuN}, \mathrm{CQ}$, and GZ. The fitting deviations are possibly due to the spatiotemporal heterogeneity of transmission parameters and detection efficiency. PRCC coefficients are used as global sensitivity to quantify the response of model outputs to the variation of the estimated parameters. By averaging the daily PRCC coefficients in the operation of fitting daily incidences, it is found that the output is strongly sensitive to the effective transmission rate of clinical infection $(\beta)$ and the relative coefficient of migration matrix $(\kappa)$, followed by the effective transmission rate of subclinical infection $(\alpha)$. Yet it seems that in the entire infection process the output is scarcely sensitive to the initial condition of the model. The reason for the negative correlation of $\beta$ and $\kappa$ with model output is that for given $R_{t}$, small values of $\beta$ and $\kappa$ mean large transmission rate $\lambda$.

In the following simulations of the model, it is set that (1) the initial conditions are $E(0)=50, I^{p}(0)=$ $I^{s}(0)=I^{c}(0)=35$ in Figs. 4,5 and $\mathrm{S} 6$, and $E(0)=I^{p}(0)=I^{s}(0)=I^{c}(0)=20$ in Figs. 6, 7 and 8 ; (2) the impact of human mobility is reflected by the migration matrix $P$, and its values are selected from Baidu migration data during 2020 and 2021; and (3) multiple interventions (including social distancing, quarantine and wearing masks) are measured by different values of the effective reproduction number $R_{t}$, in which the largest and minimum values are separately $R_{t}=3.56$ and $R_{t}=0.59$, corresponding to the situations of no intervention (in early infection stage [January 5 to January 22, 2020] in $\mathrm{HuB}$ ) and rigorous intervention (in the mid and late stage of infection [January 23 to February 12, 2020] in HuB).

Figure 4 shows the impacts of intervention on the evolution of COVID-19 in China, with different initial infection sites (i.e., HuB, BJ, SH, GD and XZ). The migration data during January 23, and March 20, 2021, are integrated into the model for simulating the transmission process under two modes: few intervention and rigorous intervention. These two modes are 
(a)

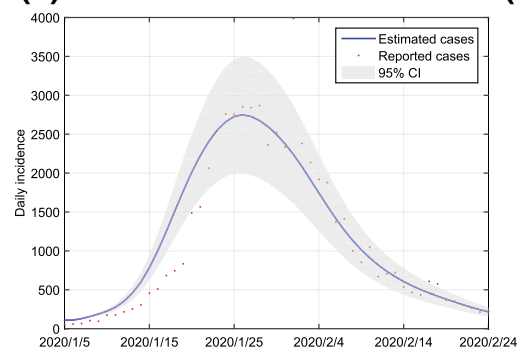

(b)

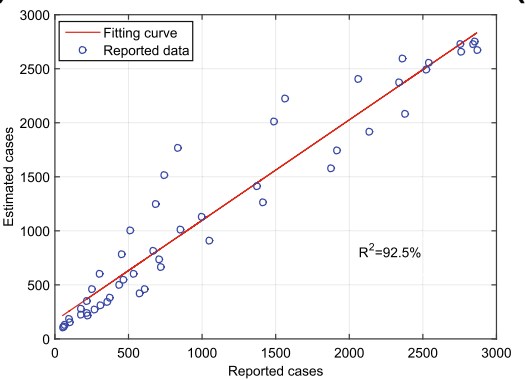

(c)

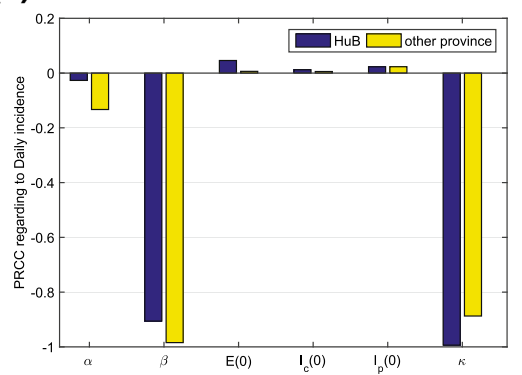

Fig. 3 The fitting results of the COVID-19 cases in China. a Fitting daily new cases in $\mathrm{HuB}$, where the light shaded area is the $95 \%$ confidence interval (CI) for all 1000 simulations, and the blue curve is the median of the model output; $\mathbf{b}$ relationship between predicted and observed cases in HuB. a sensitivity of daily cases to the model parameters as indicated by PRCC values (a)

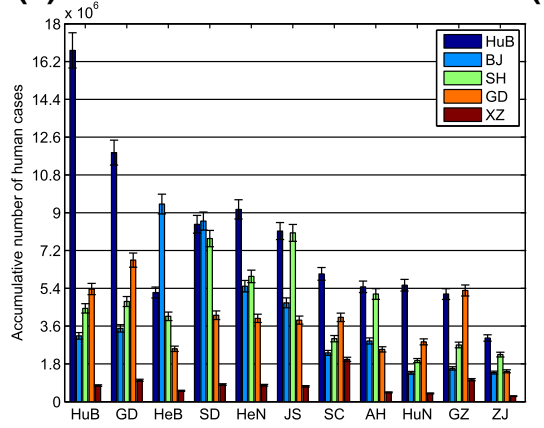

(d)

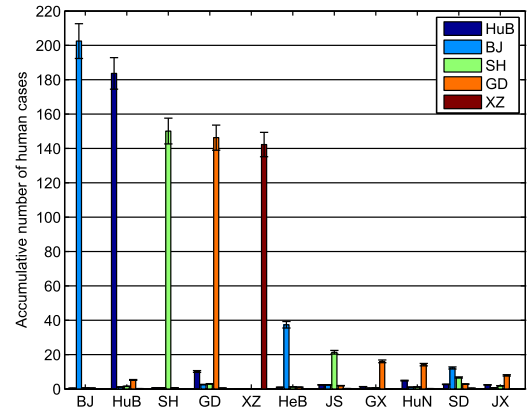

(b)

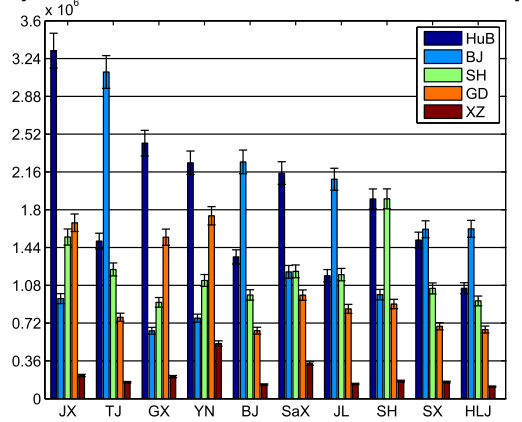

(e)

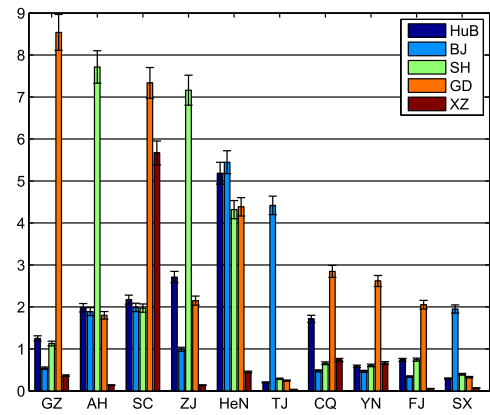

(c)

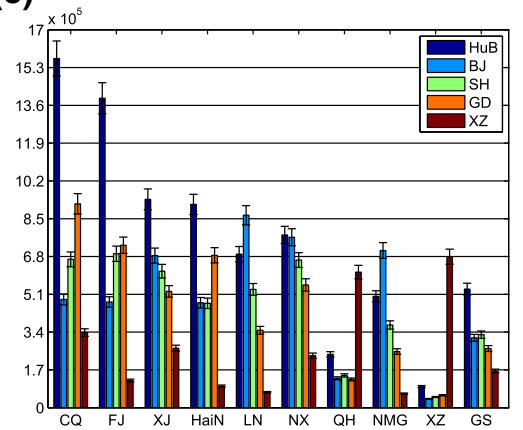

(f)

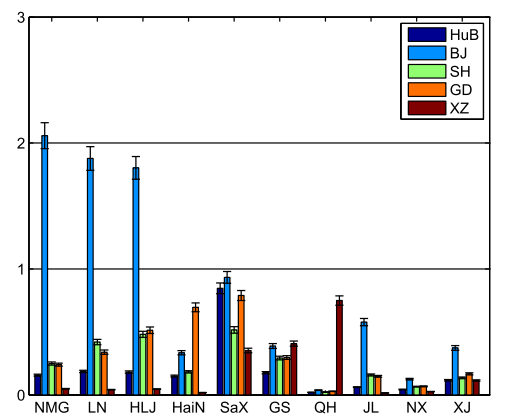

Fig. 4 The accumulative number of cases in each province before and after the intervention, in case of different locations (HuB, BJ, SH, GD, XZ) of initial infection. The human mobility information is adopted from the data during January 23 to March
20, 2021. The transmission rate $\lambda$ in Figures (a), (b), (c) and (d), (e), (f) is separately determined by the mean values of effective reproduction number before and after the intervention reflected by the choices of the reproduction number, whose values in each province are taken as the means of the effective reproduction number at the beginning of the outbreak (January 1-22, 2020) and after the intervention (January 23-February 12, 2020), respectively.
For simulating the transmission for 57 days, the following patterns are observed in Fig. 4. First, in case of substituting the early $R_{t}$ into the model, the infection burden could increase hundreds of times, in which the numbers of total clinical infections in China could 
(a)

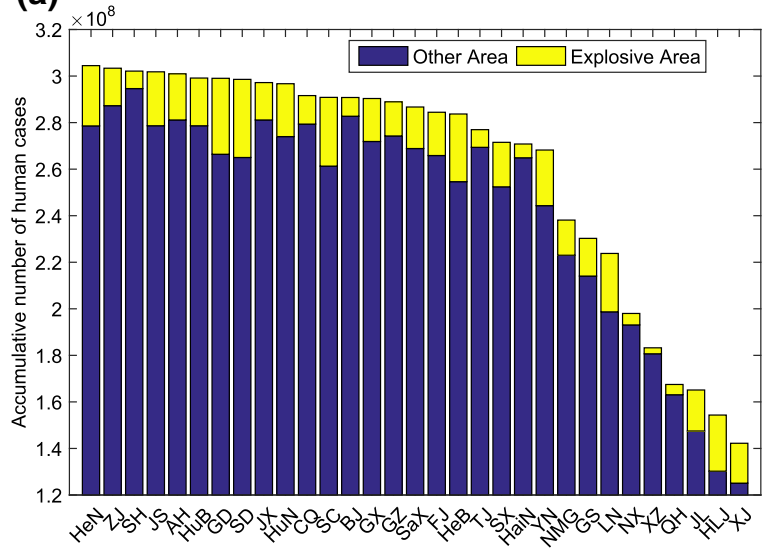

Fig. 5 The cumulative number of cases in China with the human mobility data: a from January 23 to March 20, 2021, and b from January 23 to May 20, 2021, in case of no intervention. The

reach $111.08,64.61,66.71,57.62$ and 13.59 million with separate source of initial infection in $\mathrm{HuB}, \mathrm{BJ}, \mathrm{SH}$, GD and XZ. Second, in case of substituting the latter $R_{t}$ into the model, the above numbers reduce sharply to $228,288,215,232$ and 154. Moreover, the regions around the source of initial infection would likely suffer more serious attack, in which the highest attack rates are 0.28 in $\mathrm{HuB}$ and 0.14 in GZ with source in $\mathrm{HuB}$, 0.20 in $\mathrm{TJ}$ and 0.12 in $\mathrm{HuB}$ with source in $\mathrm{BJ}, 0.10$ in JS and 0.09 in AN with source in SH, 0.15 in GZ and 0.09 in $\mathrm{HuB}$ with source in GD, 0.19 in $\mathrm{XZ}$ and 0.1 in $\mathrm{QH}$ with source in $\mathrm{XZ}$.

Figure 5 shows the ranking of total infections in China with a unique infection source at initial time and human mobility at the entire process. Here it is assumed that there is no implementation of intervention, which is realized by setting the reproduction number in each province to be the value in early infection stage in $\mathrm{HuB}$ (equal 3.56). By simulating the transmission process through 57 days, it is found that (1) the initial infection located in $\mathrm{HeN}, \mathrm{ZJ}, \mathrm{SH}$, JS and $\mathrm{AH}$ would cause the top five numbers of human clinical cases (over 300 million); (2) when the initial infection is located in XZ, $\mathrm{QH}, \mathrm{JL}, \mathrm{HLJ}, \mathrm{XJ}$, it would lead to smallest clinical infection sizes (around 142-183 million). Moreover, by simulating the transmission process through 120 days, it is observed that the infection would reach a saturated state: more than 1.1 billion people could be infected clinically, no matter where the infection ini- (b)

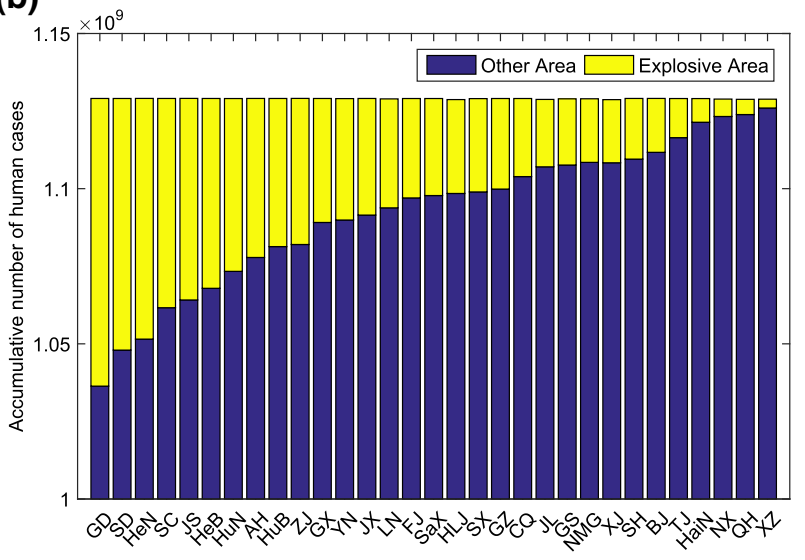

abscissa is the location that has unique infection source at the initial time. The yellow part is the contribution by the location with initial infections

tially occurs. In this case, all provinces reach the highest levels of new infections after about two months (see Fig. S3), but the attack rate exhibits spatial heterogeneity, in which the area near the initial infection source usually suffers worse.

Figure 6 shows the impacts of different initial conditions and human mobility on the evolution of COVID19 transmission across Chinese provinces, in case of no intervention. It is observed that more sites with initial infection and more frequency of human mobility would yield a little faster diffusion of the disease (that is more obvious in early infection period) and a little earlier arriving of the peak. When the disease starts to spread from January 23, 2021, the numbers of human cases would reach peak around early April or late March, in case of one initial infection site(XZ), or two initial infection sites (HeN and GD). However, in case of all sizes with the initial infection, the peak is arriving around the middle of March, regardless of population mobility. In these four settings, the serious infection would last for over three months and cause similar number of total infections (that is, 1.3 billion clinical/subclinical cases). After that the disease will still prevail in human population in very low incidence rate.

Figure 7 shows the evolution dynamics of COVID19 in China with different patterns of intervention, in case of only GD as the initial infection site. Here the impacts of intervention and human mobility are quan- 
Fig. 6 Time series of daily new cases in each province with different outbreak sites at initial time and human mobility data from January 23, 2021, to May 20, 2021, in case of no intervention

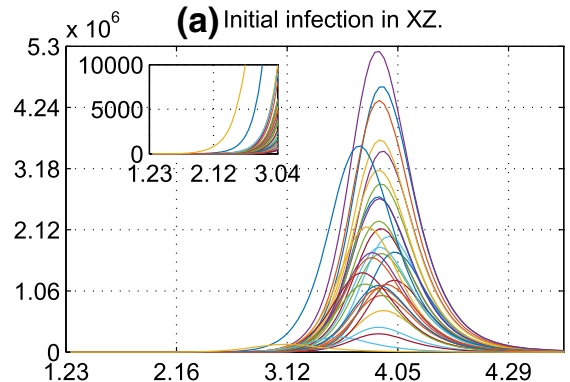

(c) Initial infection in 31 areas.

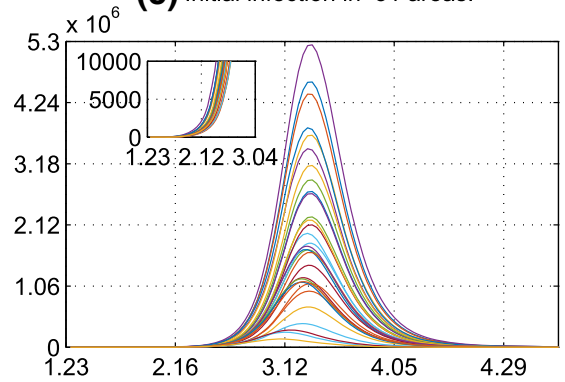

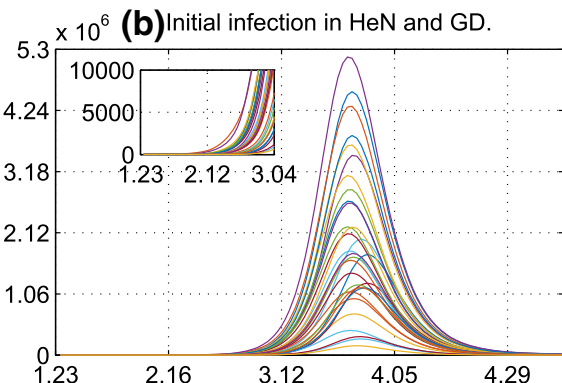

(d) Initial infection in 31 areas without movements

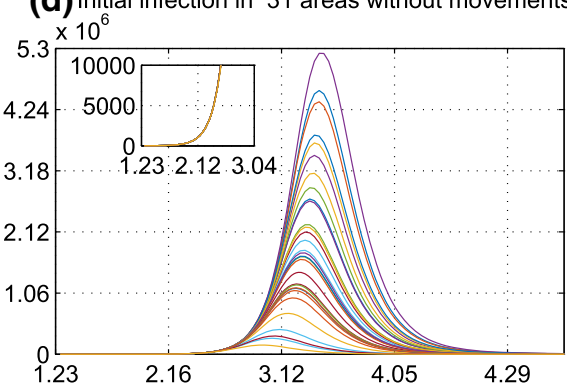

(a) Total infections in China with different interventions

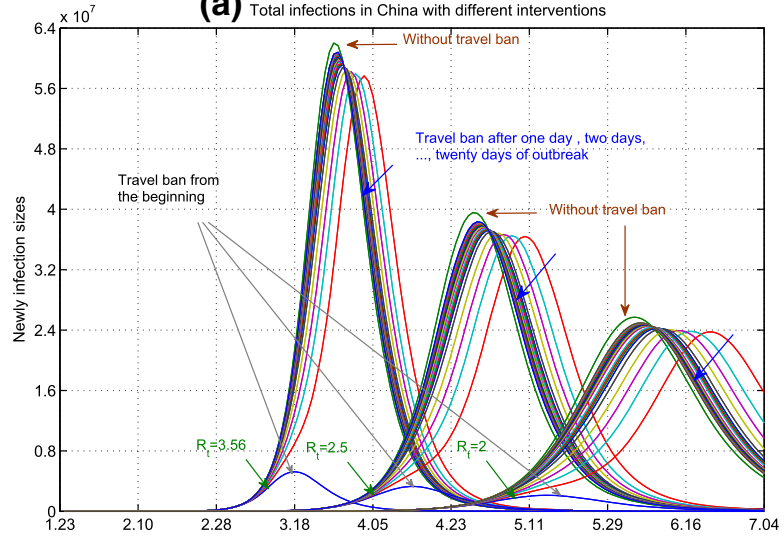

(b) Total infections in China with different interventions

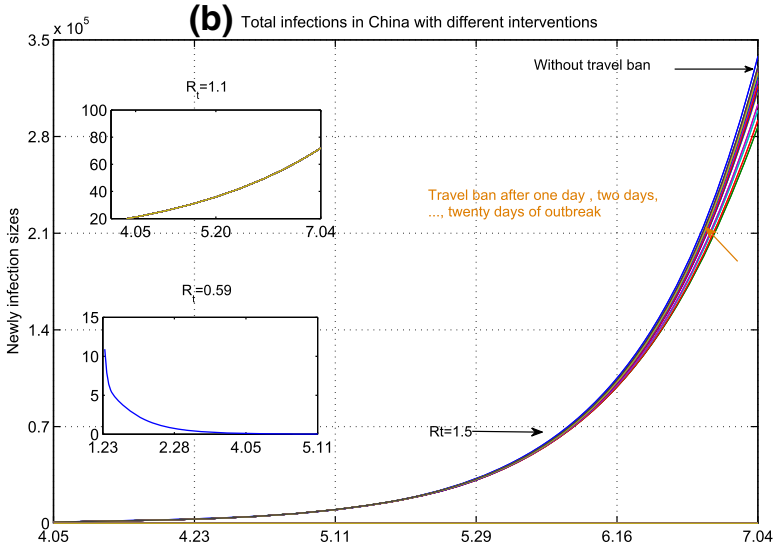

Fig. 7 Time series of daily new cases in China with different timing of travel ban and different basic reproduction number. Here human mobility data are from January 23 to July 4, 2021

tified by the basic reproduction number $R_{0}$ and travel ban, respectively. It is found that (1) slight increase of $R_{0}$ would cause rapid transmission and high morbidity around China, (2) travel ban among the provinces in China as early as possible can postpone the propagation a little bit and possibly reduce total morbidity, and (3) the control effect of travel ban is not significant (especially for large $R_{0}$ ), only when the travel is restricted at first. Specifically, by simulating the spatiotemporal transmission process for 162 days, it is observed that (1) if $R_{0}=3.56,2.5,2,1.5$, and 1.1, human infections would increase rapidly after $14,29,56,72$, and 81 days since the introduction of the infection, respectively; (2) when $R_{0}=3.56,2.5$, and 2 , the number of infections would reach the peak around March 27, May 2, and Jun 7, resulting in total clinical infections to be 1.1, 1.0 , and 0.8 billion (regardless when to start travel ban after outbreak), but the numbers would reduce vastly to $92.8,75.9$ and 85.7 million if travel ban starts before outbreak; (3) when $R_{0}=2$, if travel ban is implemented after 1 day, 10 days and 20 days of the break, the transmission could be postponed 2 day, 5 day, and 18 days (compared with the case without travel ban), resulting in $735.5,865.6$ and 876.21 million of human 

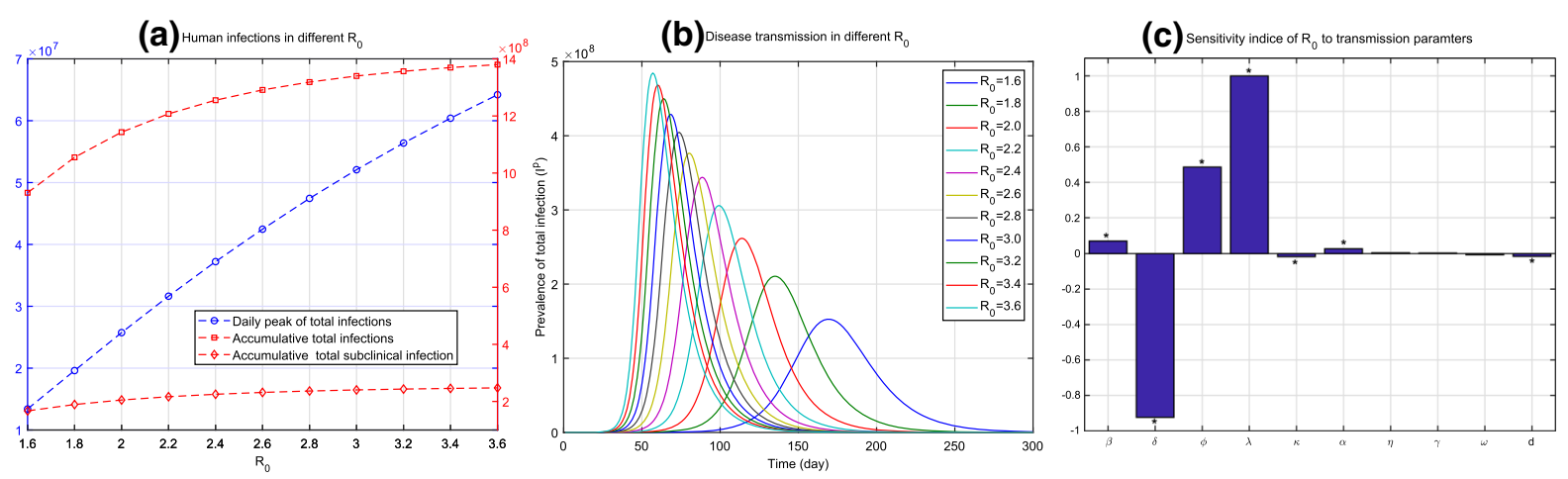

Fig. 8 Dependence of COVID-19 infection on the basic reproduction number $R_{0}$ in China (a and $\mathbf{b}$ ), and the sensitivity of $R_{0}$ to the model parameters as indicated by PRCC values. The accumulative number in $\mathbf{a}$ is the total infections during the first 400 days. Human mobility data in a and b starts on January 23, 2021, and future data are obtained by averaging those in previous time. PRCC coefficients in $\mathbf{c}$ with $*$ indicate that the corresponding parameters are significantly different from zero (with $p$-values $<0.05)$ clinical infections; and (4) in case of rigorous intervention $\left(R_{0}<1\right)$, it is impossible for travel to trigger disease outbreak.

Figure 8 shows the relationship between the basic reproduction number $R_{0}$ and human infections in China as well as the transmission parameters. It is found that the increase of $R_{0}$ easily stimulates disease prevalence and causes quick arrive and high level of the incidence peak, with a positive linear (sublinear) correlation between $R_{0}$ and the incidence peak (total infections). If $R_{0}$ increase from 2.4 to 3.2 , the accumulative number of human infections (including subclinical and clinical cases) increases from 1.26 to 1.38 billion during 400 days. Sensitivity analysis indicates that the most sensitive parameter governing $R_{0}$ is the transmission rate $\lambda$, followed by the percentage of clinical infections $(\phi)$, time span from preclinical to clinical infection $(1 / \delta)$ and the relative infectivity of clinical infection $(\beta)$.

\section{Discussion}

The COVID-19 pandemic is posing increasing threats to public health around the world. Clear information about its epidemiologic features and transmission patterns can help to control and prevent COVID-19 transmission. The present study is an attempt to provide a modeling framework allowed for inferring its spatiotemporal transmission patterns by focusing on its outbreak in the provinces of China.
Since the outbreak of COVID-19, many epidemiological models have been proposed and applied to study its propagation. Focusing on the spatiotemporal transmission, modeling framework includes mathematical model (e.g., ordinary/partial differential equation [9-12,16,17,20], difference equations [13]), computational model (e.g., agent-based model [18] and nextgeneration algorithm [19]), and statistical model (e.g., stochastic model [14] and ArcGIS [21]). Inspired by existing studies, this paper presented a new mathematical model via ODEs, which couples the intrinsic transmission dynamics, including the disease evolution in humans among different states (susceptible, exposed, infectious and recovered), infection action by humanhuman contact, and human mobility among different regions. Moreover, the effects of human behavior and control strategy were characterized by model parameters, which can regulate the spatiotemporal infectivity and transmissibility. Finally, MCMC algorithm was employed to estimate the uncertain parameters and then to evaluate the model. Here the compartmental deterministic principle used in this model is similar to those in literature $[9-14,20]$, and the spatial transmission route connecting by human mobility is consistent with epidemiological survey and related models $[15,20,28,29]$. Moreover, the model covers more transmission details, including preclinical, subclinical, and clinical infection [17,30], and can be validated by fitting multiple spatiotemporal data with fewer uncertain parameters. It captures the time-varying infectivity by 
incorporating the estimated values of the time series of the effective reproduction number, instead of formulating it by a time function. The approach technique has reference significance in the development of disease modeling.

By validating the proposed model with surveillance data in Chinese provinces, the spatiotemporal transmission dynamics and the effects of human mobility and interventions were clarified, which offers the following clues for guiding COVID-19 control.

First, there is a unique epidemic threshold, denoted by basic reproduction number $R_{0}$, which can totally determine whether COVID-19 proceeds among multiple regions. If $R_{0}<1$, no matter how many infection sources there are, COVID-19 will always die out. Otherwise, the disease will persist in each region. $R_{0}$ can unite infectivity in each region by human mobility. Such mobility contributes to transmission in two ways: susceptible persons in other regions could be infected when traveling to outbreak area, and infected persons may bring COVID-19 virus from outbreak area to other regions. Particularly, when $R_{0}>1$, no region can escape from infection if there exists human mobility among them. This $R_{0}$ is most sensitive to transmission rate $\lambda$, followed by the percentage of clinical infections. Hence reducing $\lambda$ is most effective to lessen $R_{0}$.

Second, the effects of the implemented intervention in China are further evaluated. By using the proposed model to simulate the long-term transmission process, it is found that if the interventions (e.g., social distancing and city lockdown) had not been implemented in China, COVID-19 would prevail all around China and the serious infection would last over three months, resulting in over 1.1 billion clinical patients and 0.2 billion subclinical patients. In this case, more than 92.3\% population in China would be infected clinically/subclinically by COVID-19 virus. The estimated effects of interventions are much more significant than previous results, which claimed that (1) if without nonpharmaceutical interventions in China, the number of cases was predicted to be 7.6 million by February 29, 2020 [16], or 37 million by March 5, 2020 [31], or increase the total infections by $93.7 \%$ [32]. The reason for the severity of our estimation could be that this study highlights the intrinsic spatiotemporal transmission dynamics and the total infection process.

Third, the role of human mobility in COVID-19 transmission is further clarified. Similarly to previous studies $[13,21,32]$, it is verified that human mobility (by travel) can spark new infections in virgin areas and high frequency of human mobility in reality has driven COVID-19 diffusion across the 31 provinces of China. The present paper further indicates that the effects of human mobility in the spatiotemporal transmission of COVID-19 are more prominent in two cases: early stage of infection and when $R_{0}$ is a little bigger than one. If without intervention inside region, then human mobility would accelerate disease propagation across different regions, but it could not modify the number of total infections, unless travel is banned at the very beginning of infection. Hence, regional human migration plays as a trigger in the preliminary stage of infection, and then, locally contracted infection dominates the following transmission process. The results demonstrate that non-pharmaceutical intervention is the core strategy, and travel ban at the same time can slow down the process and suppress incidence rate.

Fourth, the transmission patterns of COVID-19 in the whole country are further inferred. The initial infection located in central and east of China $(\mathrm{HeN}, \mathrm{ZJ}, \mathrm{SH}$, JS and AH) would easily stimulate quick outbreak and large infection, but adverse consequence is observed if initial infection is located in west and northeast of China (XJ, HLJ, QH, XZ, GS, NMG, and YN), in that there exists less population flow. Yet if without any intervention, the transmission would continue three months, and then, no matter where the outbreak occurs and how many sits do initial infection locate, the infections of COVID-19 would reach a saturation level, and more than $92.3 \%$ people in China would be infected. After that the incidence would keep at very low rate due to herd immunity. Yet as the increase of susceptible people, another modest wave of infection could occur after about 400 days.

In view of current situation of COVID-19 pandemic, China is facing high risk of sporadic outbreaks due to imported infections and is making great efforts for prevention. To control this disease, beside promoting vaccination (that is precisely what China is doing), the present study suggests that (1) identifying and isolating imported case is the primary mission, which can be accomplished by monitoring the travelers from foreign countries by tight and thorough surveillance system, and (2) in case of autochthonous infection, strict nonpharmaceutical interventions must be taken as soon as possible, including tracking close contacts and quarantine, travel restriction, lockdown of high risky community. Indeed, such intervention strategies are exactly as 
China is implementing. By doing so, more than $99.99 \%$ human infections would have been avoided according to this study.

Here several limitations need to be clarified. (1) The COVID-19 incidence data were based on public report information, which may yield data deviation from reality. (2) The biological parameters applied in the proposed model were extracted from the literature, which may show geographical disparities. (3) The model did not take into account the potential factors such as the difference of immunity and infectivity. Nevertheless, the model captured the dynamic evolution of disease in time and place and incorporated the biologically intuitive parameterizations. It matches well with spatiotemporal data by fitting several parameters, lending confidence to the analysis and justifying the model's further generalization.

In summary, this paper develops an inference technique for identifying the transmission patterns of COVID-19, and it is applied to explore its diffusion process in the provinces of China. The proposed model takes into account the essential effects of human mobility and disease evolution, which allow to capture the hidden spatiotemporal dynamics and internal mechanism of COVID-19 transmission. The obtained results support the interventions that are being implemented in China.

Designated parameters The bold capital letters denote $n \times 1$-dimensional column vectors. The letters with superscript as star are the expressions of the unique endemic equilibrium in the model. Number $\mathbf{0}$ and letter O separately represent a $n \times 1$ column vector and a $n \times n$ matrix, each element of which is zero. The Chinese provinces and their abbreviations are shown in Table 2.

Acknowledgements This work was supported by the National Natural Science Foundation of China (82041021) and the Innovation Project of GUET Graduate Education (2021YCXS111 and 2020YCXS085).

Author contributions HL and GZ designed the study. QJ, JL, HL, and FT collected the data. QJ, JL, HL, and GZ built the model and performed the analysis. QJ, JL, and FT interpreted the data. QJ and GZ prepared the manuscript. All authors reviewed and approved the submitted manuscript.
Data availibility The data that support the findings of this study are available from the corresponding author upon reasonable request.

\section{Declarations}

Conflict of interest The authors declare no conflict of interest.

\section{References}

1. World Health Organization (WHO): Coronavirus disease (COVID-19) pandemic. https://www.who.int/zh/ emergencies/diseases/novel-coronavirus (2019)

2. CDC.: Epidemic update and risk assessment of 2019 Novel Coronavirus

3. Cuadros, D.F., Xiao, Y., Mukandavire, Z., Correa-Agudelo, E., Hernández, A., Kim, H., et al.: Spatiotemporal transmission dynamics of the COVID-19 pandemic and its impact on critical healthcare capacity. Health Place 64, 102404 (2020)

4. Shereen, M.A., Khan, S., Kazmi, A., Bashir, N., Siddique, R.: COVID-19 infection: origin, transmission, and characteristics of human coronaviruses. J. Adv. Res. 24, 91-98 (2020)

5. Gao, M., Yang, L., Chen, X., Deng, Y., Yang, S., Xu, H., et al.: A study on infectivity of asymptomatic SARS-CoV-2 carriers. Respir. Med. 169, 106026 (2020)

6. Nishiura, H., Linton, N.M., Akhmetzhanov, A.R.: Serial interval of novel coronavirus (COVID-19) infections. Int. J. Infect. Dis. 93, 284-286 (2020)

7. Godri Pollitt, K.J., Peccia, J., Ko, A.I., Kaminski, N., Dela Cruz, C.S., Nebert, D.W., et al.: COVID-19 vulnerability: the potential impact of genetic susceptibility and airborne transmission. Human Genom. 14, 1-7 (2020)

8. Kamp, C.: Untangling the interplay between epidemic spread and transmission network dynamics. PLoS Comput. Biol. 6(11), e1000984 (2010)

9. He, S., Peng, Y., Sun, K.: SEIR modeling of the COVID-19 and its dynamics. Nonlinear Dyn. 101(3), 1667-1680 (2020)

10. Hou, C., Chen, J., Zhou, Y., Hua, L., Yuan, J., He, S., et al.: The effectiveness of quarantine of Wuhan city against the Corona Virus Disease 2019 (COVID-19): a well-mixed SEIR model analysis. J. Med. Virol. 92(7), 841-848 (2020)

11. Yang, Z., Zeng, Z., Wang, K., Wong, S., Liang, W., Zanin, M., et al.: Modified SEIR and AI prediction of the epidemics trend of COVID-19 in China under public health interventions. J. Thoracic Dis. 12(3), 165-174 (2020)

12. Viguerie, A., Lorenzo, G., Auricchio, F., Baroli, D., Hughes, T.J., Patton, A., et al.: Simulating the spread of COVID19 via a spatially-resolved susceptible-exposed-infectedrecovered-deceased (SEIRD) model with heterogeneous diffusion. Appl. Math. Lett. 111, 106617 (2021)

13. Prem, K., Liu, Y., Russell, T.W., Kucharski, A.J., Eggo, R.M., Davies, N., et al.: The effect of control strategies to reduce social mixing on outcomes of the COVID-19 epidemic in Wuhan, China: a modelling study. Lancet Public Health 5(5), e261-e270 (2020)

14. Hou, X., Gao, S., Li, Q., Kang, Y., Chen, N., Chen, K., et al.: Intracounty modeling of COVID-19 infection with human 
mobility: assessing spatial heterogeneity with business traffic, age, and race. Proc. Natl. Acad. Sci. 118(24), (2021)

15. Kraemer, M.U., Yang, C., Gutierrez, B., Wu, C., Klein, B., Pigott, D.M., et al.: The effect of human mobility and control measures on the COVID-19 epidemic in China. Science 368(6490), 493-497 (2020)

16. Lai, S., Ruktanonchai, N.W., Zhou, L., Prosper, O., Luo, W., Floyd, J.R., et al.: Effect of non-pharmaceutical interventions to contain COVID-19 in China. Nature 585(7825), 410-413 (2020)

17. Davies, N.G., Kucharski, A.J., Eggo, R.M., Gimma, A., Edmunds, W.J., Jombart, T., et al.: Effects of nonpharmaceutical interventions on COVID-19 cases, deaths, and demand for hospital services in the UK: a modelling study. Lancet Public Health 5(7), e375-e385 (2020)

18. Cuevas, E.: An agent-based model to evaluate the COVID19 transmission risks in facilities. Comput. Biol. Med. 121, 103827 (2020)

19. Liu, Y., Gu, Z., Xia, S., Shi, B., Zhou, X., Shi, Y., et al.: What are the underlying transmission patterns of COVID-19 outbreak? An age-specific social contact characterization. EClinicalMedicine 22, 100354 (2020)

20. Linka, K., Peirlinck, M., Sahli Costabal, F., Kuhl, E.: Outbreak dynamics of COVID-19 in Europe and the effect of travel restrictions. Comput. Methods Biomech. Biomed. Eng. 23(11), 710-717 (2020)

21. Kang, D., Choi, H., Kim, J., Choi, J.: Spatial epidemic dynamics of the COVID-19 outbreak in China. Int. J. Infect. Dis. 94, 96-102 (2020)

22. Mizumoto, K., Kagaya, K., Zarebski, A., Chowell, G.: Estimating the asymptomatic proportion of coronavirus disease 2019 (COVID-19) cases on board the Diamond Princess cruise ship, Yokohama, Japan, 2020. Eurosurveillance 25(10), 2000180 (2020)

23. Van den Driessche, P., Watmough, J.: Reproduction numbers and sub-threshold endemic equilibria for compartmental models of disease transmission. Math. Biosci. 180(1-2), 29-48 (2002)

24. Shuai, Z., van den Driessche, P.: Global stability of infectious disease models using Lyapunov functions. SIAM J. Appl. Math. 73(4), 1513-1532 (2013)
25. Bessey, K., Mavis, M., Rebaza, J., Zhang, J.: Global stability analysis of a general model of Zika virus. Nonauton. Dyn. Syst. 6(1), 18-34 (2019)

26. Thompson, R., Stockwin, J., van Gaalen, R.D., Polonsky, J., Kamvar, Z., Demarsh, P., et al.: Improved inference of time-varying reproduction numbers during infectious disease outbreaks. Epidemics 29, 100356 (2019)

27. Li, Q., Guan, X., Wu, P., Wang, X., Zhou, L., Tong, Y., et al.: Early transmission dynamics in Wuhan, China, of novel coronavirus-infected pneumonia. N. Engl. J. Med. 382(13), (2020)

28. Badr, H.S., Du, H., Marshall, M., Dong, E., Squire, M.M., Gardner, L.M.: Association between mobility patterns and covid-19 transmission in the USA: a mathematical modelling study. Lancet Infect. Dis. 20(11), 1247-1254 (2020)

29. Chang, S., Pierson, E., Koh, P.W., Gerardin, J., Redbird, B., Grusky, D., et al.: Mobility network models of covid-19 explain inequities and inform reopening. Nature 589(7840), 82-87 (2021)

30. Davies, N.G., Klepac, P., Liu, Y., Prem, K., Jit, M., Eggo, R.M.: Age-dependent effects in the transmission and control of covid-19 epidemics. Nat. Med. 26(8), 1205-1211 (2020)

31. Hsiang, S., Allen, D., Annan-Phan, S., Bell, K., Bolliger, I., Chong, T., et al.: The effect of large-scale anti-contagion policies on the COVID-19 pandemic. Nature 584(7820), 262-267 (2020)

32. Zhang, B., Liang, S., Wang, G., Zhang, C., Chen, C., Zou, M., et al.: Synchronized nonpharmaceutical interventions for the control of COVID-19. Nonlinear Dyn. (2021). https:// doi.org/10.1007/s11071-021-06505-0

Publisher's Note Springer Nature remains neutral with regard to jurisdictional claims in published maps and institutional affiliations. 